\title{
Creep Behavior of Passive Bovine Extraocular Muscle
}

\author{
Lawrence Yoo, ${ }^{1,2}$ Hansang Kim, ${ }^{2}$ Andrew Shin, ${ }^{1,2}$ Vijay Gupta, ${ }^{2,3}$ and Joseph L. Demer ${ }^{1,3,4,5}$ \\ ${ }^{1}$ Department of Ophthalmology, Jules Stein Eye Institute, University of California Los Angeles, 100 Stein Plaza, UCLA, \\ Los Angeles, CA 90095-7002, USA \\ ${ }^{2}$ Department of Mechanical Engineering, University of California, Los Angeles, CA, USA \\ ${ }^{3}$ Biomedical Engineering Interdepartmental Program, University of California, Los Angeles, CA, USA \\ ${ }^{4}$ Neuroscience Interdepartmental Program, University of California, Los Angeles, CA, USA \\ ${ }^{5}$ Department of Neurology, University of California, Los Angeles, CA, USA
}

Correspondence should be addressed to Joseph L. Demer, jld@ucla.edu

Received 6 June 2011; Accepted 16 August 2011

Academic Editor: Henk Granzier

Copyright () 2011 Lawrence Yoo et al. This is an open access article distributed under the Creative Commons Attribution License, which permits unrestricted use, distribution, and reproduction in any medium, provided the original work is properly cited.

This paper characterized bovine extraocular muscles (EOMs) using creep, which represents long-term stretching induced by a constant force. After preliminary optimization of testing conditions, 20 fresh EOM samples were subjected to four different loading rates of $1.67,3.33,8.33$, and $16.67 \% / \mathrm{s}$, after which creep was observed for 1,500 s. A published quasilinear viscoelastic (QLV) relaxation function was transformed to a creep function that was compared with data. Repeatable creep was observed for each loading rate and was similar among all six anatomical EOMs. The mean creep coefficient after 1,500 seconds for a wide range of initial loading rates was at $1.37 \pm 0.03$ (standard deviation, SD). The creep function derived from the relaxation-based QLV model agreed with observed creep to within 2.7\% following 16.67\%/s ramp loading. Measured creep agrees closely with a derived QLV model of EOM relaxation, validating a previous QLV model for characterization of EOM biomechanics.

\section{Introduction}

Since strabismus surgery manipulates extraocular muscles (EOMs) mechanically to correct binocular misalignment, accurate determination of quasistatic biomechanical properties of EOMs may be important. Classical studies investigated the uniaxial force and length relationship for EOMs [1-4]. More recently, comprehensive biomechanical methods have been employed to characterize constitutive models [5] for EOMs, representing their viscoelastic, or history-dependent relationship between stress and strain $[6,7]$. Several important mechanical behaviors reflect viscoelasticity: hysteresis, relaxation, attenuation of acoustic waves, and creep.

Hysteresis is history dependent variation in mechanical behavior, resulting in differences in stress between loading and unloading phases. Hysteresis implies work, energy dissipation due to loading and unloading $[5,8,9]$. Relaxation describes how a material deformed by external perturbation returns to equilibrium. In stress relaxation testing, a material is rapidly subjected to displacement and then maintained over an extended time interval at the same displacement by external feedback while a decline in required external force is observed $[6,10-13]$.

Creep is the tendency of a material to deform permanently under constant force. To characterize creep, tensile loading is rapidly imposed and maintained at a constant level while specimen elongation is observed over an extended time $[13,14]$. Creep is likely to be particularly significant in binocular alignment and strabismus, where agonist and antagonist EOMs remain under loading for extended time periods, and where such loading is purposely altered by, for example, strabismus surgery that alters EOM tension.

While viscoelastic properties have often been neglected in interpretation of conventional EOM length-tension data, realistic constitutive modeling is essential for accurate finite element models (FEMs) that graphically simulate interactions of EOMs with orbital connective tissues. Supporting this contention, Quaia et al. [7] and our previous investigation [6] highlighted the need for valid constitutive models of EOMs in application of quasilinear viscoelastic (QLV) theory to constitutive modeling. 
Linear elasticity theory is well established for strains of $\leq 3 \%$ in generic materials. Soft tissues generally, however, undergo larger physiological strains in the range of tens of percent where behavior becomes nonlinear. Modeling of EOMs should consider the hyperelastic nature of soft tissue, which means that it is highly nonlinearly elastic $[12,15-$ 20] Fung's QLV theory [5, 21-23] is appropriate because it incorporates both linear viscoelastic and nonlinear elastic characteristics. We previously reported that a QLV model based upon in vitro relaxation data was effective in describing mechanical behavior of passive bovine EOMs [6].

Quaia et al. found limitations in QLV describing constitutive properties of in vivo simian EOMs at short-time scale and proposed a modification that they termed adaptive quasilinear viscoelasticity (AQLV) [7]. Although AQLV was superior to QLV in describing EOM properties relevant to the most rapid eye movements (saccades), the approach is computationally intensive and requires extensive data collection that would limit its practicality for FEM. We supposed that the simpler QLV formulation might be adequate to quasistatic, passive behavior of EOMs relevant in binocular alignment and strabismus.

Since the EOMs are not contracting when under tensile loading, we emulated a previous study with the assumption that absence of perfusion and innervation would not strongly influence constitutive properties [6]. By investigating EOM creep, we aimed to extend understanding of their timedependent stress-strain behavior and to validate the previously reported relaxation-based QLV model applicable to FEM [6].

\section{Methods}

The approach was similar to that previously published [6]. The EOM specimens prepared from heads of cattle freshly slaughtered for food. The total time from slaughter to mechanical testing ranged from 3 to 4 hours. During the preparation of specimens, the tissues were constantly irrigated with lactated Ringer solution, which was kept at $37^{\circ} \mathrm{C}$, to prevent any dehydration. The EOM specimens were cut into $2 \sim 3 \mathrm{~cm}$ long rectangular prisms (length of actual bovine EOMs) having $2 \mathrm{~mm} \times 2 \mathrm{~mm}$ cross-section. Actual test length of each specimen was $10 \mathrm{~mm}$, with $\leq 5 \mathrm{~mm}$ on both ends that was clamped on the load cell, excluding the terminal tendon. As in the previous study, a tabletop microtensile load cell, Instron model 4411 (Instron, Norwood, Mass, Series IX software) was enclosed in a plastic chamber where warm water vapor and radiant heat maintained physiologic $97 \%$ humidity and $37^{\circ} \mathrm{C}$ temperature as indicated by a sensor (Fisher Scientific, Chino, Calif). Preconditioning was omitted because a previous investigation of passive EOM relaxation properties demonstrated that preconditioning did not influence results [6]. For both creep testing and preliminary experiments to optimize the testing parameters, total of 60 EOM specimens from 8 different bovine orbits were used.

2.1. Creep Testing. We determined the reduced creep $(\varepsilon(t) /$ $\varepsilon(0)$ ). In ideal creep testing, an initial tensile force should be imposed on the specimen instantaneously and then maintained at a constant level for an extended time $[5,13]$. Since instantaneous imposition of tensile force is physically impossible, tensile force is rapidly increased at a constant ramp rate to a level that is subsequently maintained. Based upon tensile-loading results [6], we selected $0.2 \mathrm{~N}$, which produces $30 \%$ strain that is well within the linear range, as an appropriate ramp force. Although the maximum loading rate of the load cell was $550 \mathrm{~mm} / \mathrm{min}$, it was necessary to limit the rate to $100 \mathrm{~mm} / \mathrm{min}$ to maintain stable force feedback. Therefore, all the creep tests were performed at the following four rates: $10,20,50$, and $100 \mathrm{~mm} / \mathrm{min}$, equivalent to 1.67 , $3.33,8.33$, and $16.67 \% / \mathrm{s}$, respectively. After the initial loading of $0.2 \mathrm{~N}$ was imposed, this force was then maintained by the load cell's feedback servo for $1,500 \mathrm{~s}$ during recording of specimen length.

2.2. Fung's Quasilinear Viscoelasticity Theory. It represents stress as a function of strain and time. In stress relaxation testing, strain is held constant and the stress declines as a function of time alone:

$$
\sigma(\mathbf{t})=\sigma_{0} \mathrm{G}(\mathbf{t}),
$$

where $\mathbf{G}(\mathbf{t})$ is the time dependent reduced relaxation function with $\mathbf{G}(\mathbf{0})=\mathbf{1}$, and $\sigma_{0}$ is initial stress. Similarly, for static creep in which stress is held constant, strain varies as a function of time alone:

$$
\boldsymbol{\varepsilon}(\mathbf{t})=\varepsilon_{0} \mathbf{J}(\mathbf{t}),
$$

where $\mathbf{J}(\mathbf{t})$ is the time dependent reduced creep function with $\mathbf{J}(\mathbf{0})=\mathbf{1}$ and $\boldsymbol{\varepsilon}_{0}$ is the initial strain. For linear viscoelasticity [5], which is a special case of the more general QLV theory [24], time-varying stress relaxation function $\mathbf{G}(\mathbf{t})$ and creep function $\mathbf{J}(\mathbf{t})$ should be related as follows:

$$
\begin{aligned}
\mathbf{G}(0) & =\frac{1}{\mathbf{J}(\mathbf{0})}, \\
\mathbf{G}(\infty) & =\frac{1}{\mathrm{~J}(\infty)},
\end{aligned}
$$

or

$$
\mathrm{G}(\mathbf{s}) \mathbf{J}(\mathbf{s})=\frac{1}{\mathbf{s}^{2}},
$$

where $\mathbf{G}(\mathbf{s})$ and $\mathbf{J}(\mathbf{s})$ are Laplace transforms of the functions $\mathbf{G}(\mathbf{t})$ and $\mathbf{J}(\mathbf{t})$, respectively. A nonlinear least square method was used in previous study fit the stress relaxation data, determining constants $\mathbf{a}, \mathbf{b}, \mathbf{c}, \mathbf{d}, \mathbf{g}$, and $\mathbf{h}[6]$ :

$$
\mathrm{G}(\mathrm{t})=\mathbf{a} \mathrm{e}^{(-\mathrm{b} t)}+\mathbf{c e} \mathrm{e}^{(-\mathrm{d} t)}+\mathbf{g e} \mathrm{e}^{(-\mathrm{ht})} .
$$

Using the Laplace transform of the stress relaxation function and (4), a predicted creep function for EOM was determined using MatLab (The Mathworks Inc, Natick, Mass, USA).

\section{Results and Discussion}

As is typical for creep testing generally $[5,24,25]$, a logarithmically increasing displacement was observed after 


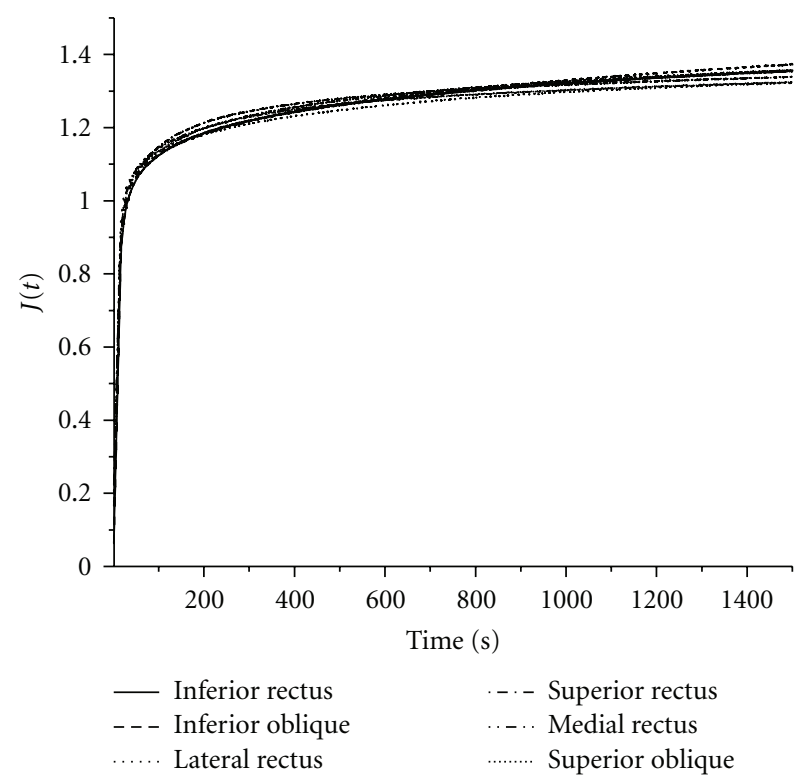

(a)

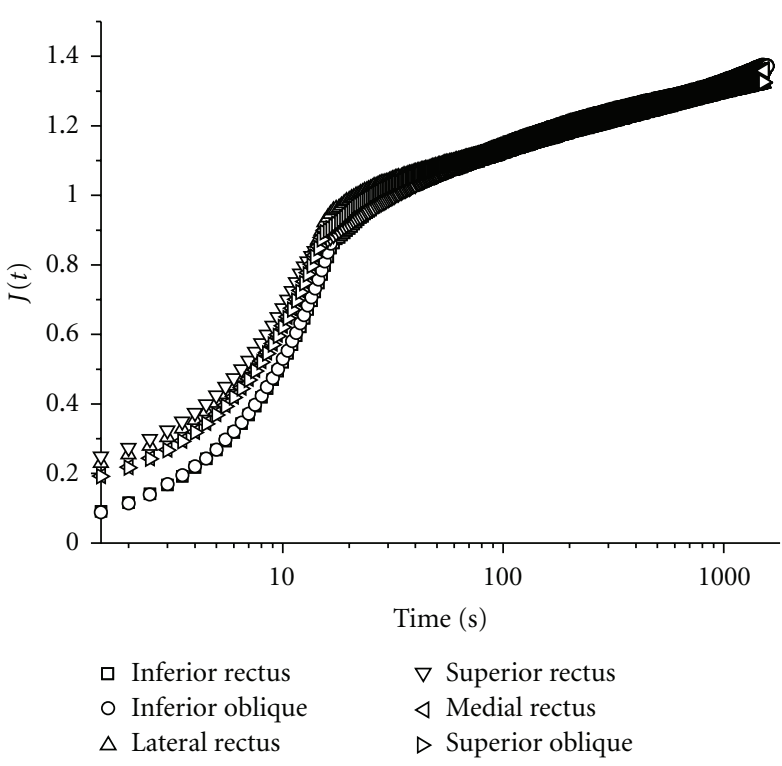

(b)

FIGURE 1: Mean normalized displacement $J(t)$ plotted for two specimens each of the six bovine EOMs in both linear and semi-log scales. All EOMs exhibited similar creep to $1.34 \pm 0.02(\mathrm{SD})$ maximum.

initial ramp loading of each specimen, without asymptote. Data were plotted as normalized displacement $J(t)$, the ratio of instantaneous specimen length to initial length.

3.1. Creep for Each Anatomical EOM. Although relaxation and tensile properties were similar among the four rectus and both oblique bovine EOMs in the previous study [6], preliminary experiments were conducted on two specimens of each of the six EOMs to determine if they exhibit similar creep at $3.33 \% / s$ strain rate. As can be seen from Figure 1, creep was essentially identical for all six EOMs.

3.2. Dependence of Creep on Ramp-Loading Rate. In order to determine if EOM properties depend on initial loading, experiments were conducted for five specimens each at four ramp-loading rates: $1.67,3.33,8.33$, and $16.67 \% / \mathrm{s}$. As an example, Figures 2(a) and 2(b) show the force displacement and creep plots for $0.2 \mathrm{~N}$ force. Since all EOMs exhibited similar creep, differentiation of each anatomical EOM was unnecessary.

Creep at other ramp-loading rates was similar to Figure 2, with maximum standard deviations (SDs) for five specimens each of $0.029,0.056$, and 0.036 for $3.33,8.33$, and $16.67 \% / s$ loading, respectively.

As seen in Figure 3, for all four initial loading rates, creep coefficients at the end of 1,500 seconds were similar at $1.37 \pm 0.033$. Despite the ultimate similarity of creep behavior, the linear part of each curve under initial loading steepened as loading rate increased, and the shape of each curve varied with loading rate (Figure 3). As loading rate decreased, the curve showed slower elongation. Since EOM is a soft tissue,
TABLE 1: QLV relaxation model parameters.

\begin{tabular}{lc}
\hline$a$ & 2.81 \\
$b(1 / \mathrm{sec})$ & 1.57 \\
$c$ & 0.86 \\
$d(1 / \mathrm{sec})$ & $1.4 \times 10^{-4}$ \\
$g$ & 0.34 \\
$h(1 / \mathrm{sec})$ & $1.7 \times 10^{-2}$ \\
\hline
\end{tabular}

reaching a true asymptotic value during creep testing is neither practical nor theoretically necessary $[5,25]$. Although the creeping behavior exhibited by EOM after ramp loading is used in its viscoelastic characterization, the rate-dependent ramp behavior can also be used to understand the contribution of EOM elasticity in its overall creep behavior. With behavior of the EOM during the ramp phase being less affected by viscosity as the loading rate is decreased, one can optimize the loading rate for tensile testing to extract the tensile elasticity of the EOM.

3.3. Conversion of Relaxation Function to Creep Function. All constants in the reduced relaxation function were derived from the published relaxation function [6] and are shown in Table 1.

Using parameters extracted from the QLV relaxation model, (4) in the time domain was converted to a reduced creep function using the inverse Laplace transform function and symbolic toolbox in Matlab, 6 constant values from the Laplace $S$ domain (6), and 7 constants from the time 


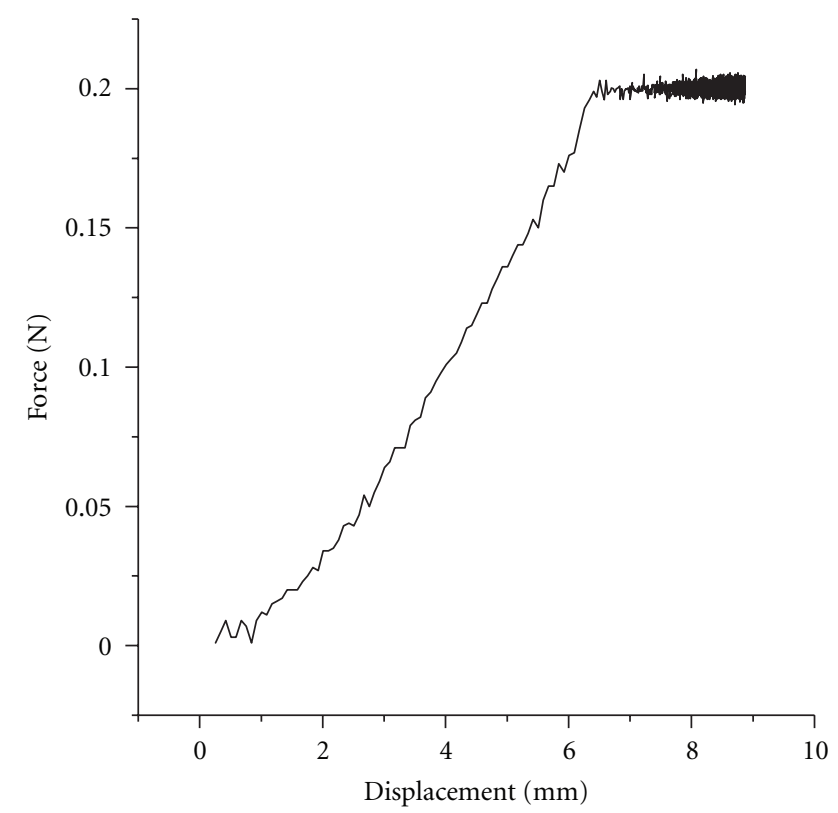

(a)

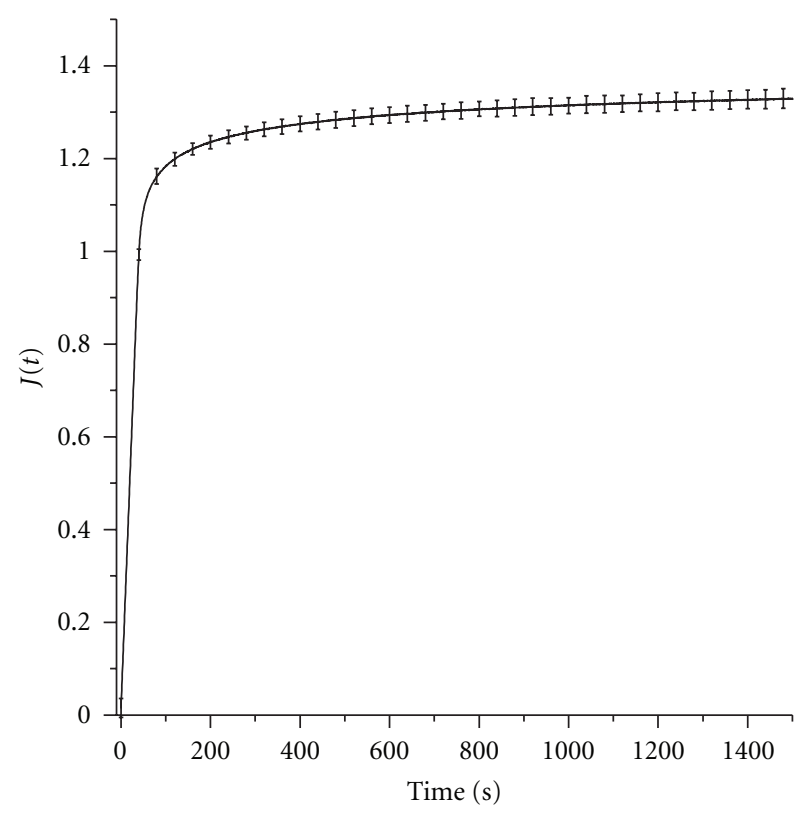

(b)

FIgURE 2: Mean creep for 1.67\%/s loading rate for 5 EOM specimens. (a) Force versus displacement. After initial ramp loading achieved at around $6 \mathrm{~mm}$ deformation, the force was held constant at $0.2 \mathrm{~N}$. (b) Reduced creep coefficients over 1,500 seconds. Maximum creep coefficient SD was 0.023 .

TABle 2: Constants in Time and Laplace $S$ Domain for Creep (6) and (7).

\begin{tabular}{lclc}
\hline$a_{1}$ & 2.81 & $C_{1}$ & $1.6137 \times 10^{-4}$ \\
$a_{2}$ & 1.57 & $C_{2}$ & $4.0473 \times 10^{21}$ \\
$a_{3}$ & 0.86 & $C_{3}$ & 0.2312 \\
$a_{4}$ & $1.4 \times 10^{-4}$ & $C_{4}$ & $1.5097 \times 10^{22}$ \\
$a_{5}$ & 0.34 & $C_{5}$ & $1.6788 \times 10^{22}$ \\
$a_{6}$ & $1.7 \times 10^{-2}$ & $C_{6}$ & 0.2434 \\
& & $C_{7}$ & 1.1487 \\
\hline
\end{tabular}

domain (7) of the resulting reduced creep function are listed in Table 2

$$
\begin{gathered}
J(s)=\frac{1}{S^{2}\left(a_{1} /\left(s+a_{2}\right)+a_{3} /\left(s+a_{4}\right)+a_{5} /\left(s+a_{6}\right)\right)}, \\
J(t)=C_{1} t+\frac{C_{2} \sinh \left(C_{3} t\right)-C_{4} \cosh \left(C_{3} t\right)}{C_{5} \exp \left(C_{6} t\right)}+C_{7} .
\end{gathered}
$$

Since ideal initial loading is assumed instantaneous, data obtained at higher rates of initial ramp loading should more closely reflect ideal creep. Hence, the reduced creep function derived from the reduced relaxation function was compared with experimental data for the highest ramp-loading rate, showing close agreement (Figure 4). Experimental creep coefficient values fell within $\pm 2.7 \%$ of theoretical values for all time points tested.

When an EOM is innervated and perfused with oxygenated blood, it is constantly under tension. It is important to understand EOM creep properties for simulation of the

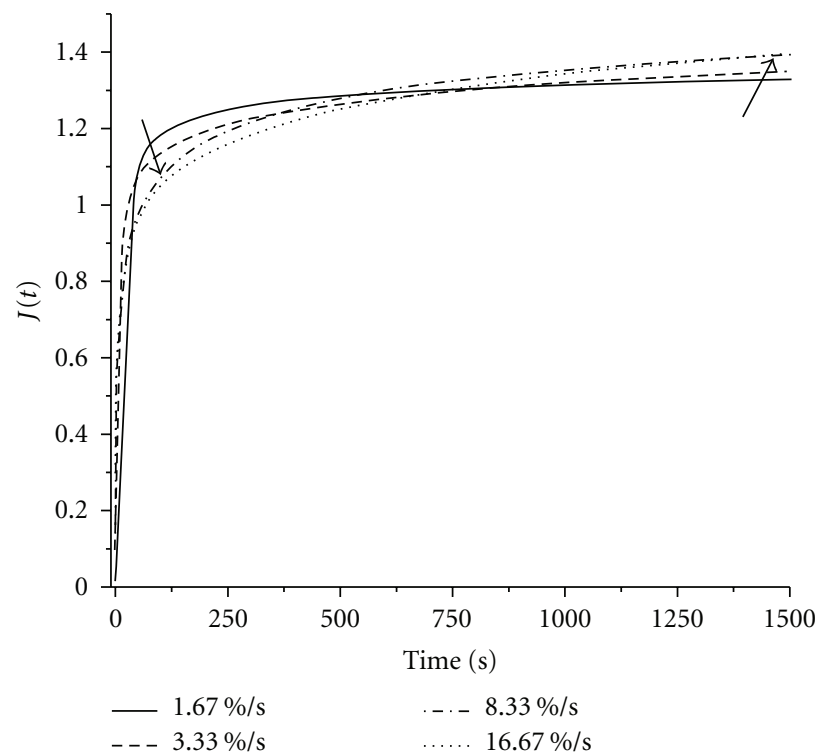

FIgURE 3: Comparison of creep at 4 different initial loading rates. The left arrow indicates that a lower reduced creep coefficient is reached at the end of initial ramp loading as the ramp-loading rate increases. The right arrow indicates that a higher creep coefficient is reached after 1500 seconds as ramp-loading rate increases.

common situation where a physiologically relaxing EOM is still experiencing tensile force exerted by its antagonist. In the current study, passive bovine EOM creep was investigated within the framework of linear viscoelasticity by comparing converted creep function from QLV model based upon stress 


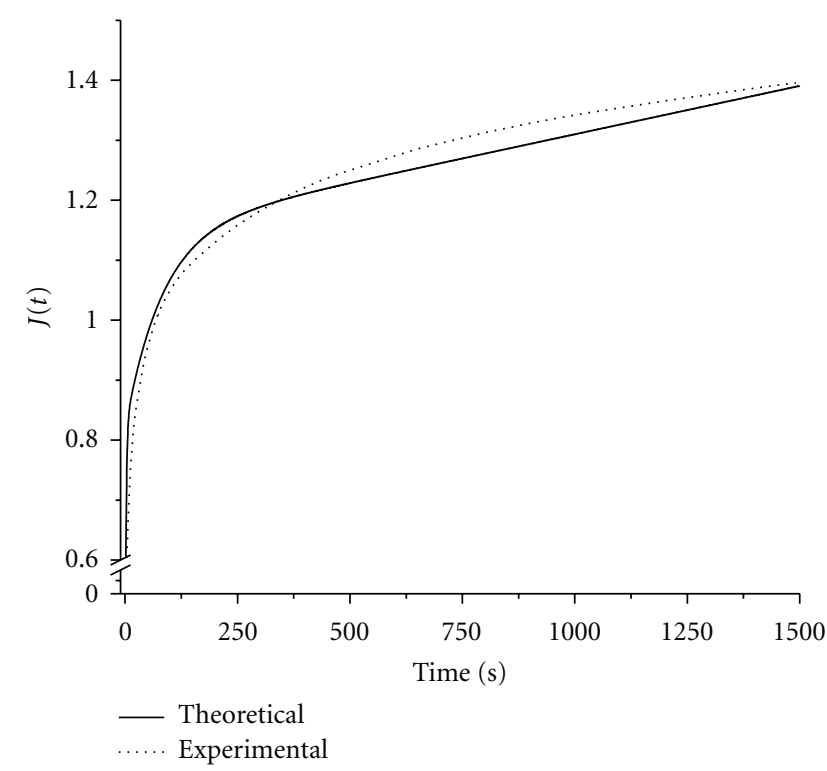

FIgURE 4: Observed reduced EOM creep function, and theoretical function derived from the reduced relaxation function.

relaxation data [6]. The current experiment varied initial elongation rate and demonstrated excellent agreement with the theoretical prediction based upon QLV relaxation model. It has been suggested that initial ramp loading for creep testing of articular cartilage should occur within $250 \mathrm{~ms}$ [20]; however, even over a longer time of $800 \mathrm{~ms}$, the data for bovine EOMs agreed closely with values derived from relaxation function.

Regardless of which anatomical rectus or oblique EOM was tested, creep for initial ramp-loading rates from 1.67 to $16.7 \% / \mathrm{s}$ showed similar asymptotic creep coefficients of $1.37 \pm 0.03$ over $1,500 \mathrm{~s}$. However, dynamic creep varied with initial strain rate. Creep coefficient $J(t)$ reached a higher initial value when the loading rate was lower, but subsequently increased more slowly (Figure 3). It seems reasonable to assume that each EOM specimen has the same elasticity. It is a well-accepted notion that when solid specimen is stretched slowly, dynamic contributions are reduced because at low initial loading speed, elasticity predominates over viscous effects [26-28]. With predominantly elastic effects, less energy is dissipated than when initial loading is rapid. Since the present models were based upon data from low strain rates, we suggest that the QLV models derived from these investigations are most applicable to slow eye movements such as fixations and pursuit. Since loading for both relaxation and creep tests closely approximates ideal step loading, the relaxation and creep functions are likely to accurately reflect EOM viscosity.

\section{Conclusion}

This paper validated a previously reported QLV model for EOM relaxation. Since a reduced creep function is the inverse Laplace transform of a reduced relaxation function, agreement between experimental creep and the previously published reduced relaxation function constitutes a strong test of the relaxation-based QLV model for passive EOMs, with experimental agreement to within $\pm 2.7 \%$ of theoretical values. Hence, we can infer that a QLV model based upon relaxation effectively describes the constitutive properties of passive EOMs. The present validation of the quantitative viscoelastic constitutive relationship for passive bovine EOM provides better understanding of EOM biomechanics, in a theoretical framework practical for graphical simulation of quasistatic ocular motility using FEM.

\section{Acknowledgments}

This paper is supported by US Public Health Service, National Eye Institute: Grants EY08313 and EY00331; Research to Prevent Blindness. J. Demer is Leonard Apt Professor of Ophthalmology.

\section{References}

[1] D. A. Robinson, D. M. O'Meara, A. B. Scott, and C. C. Collins, "Mechanical components of human eye movements," Journal of Applied Physiology, vol. 26, no. 5, pp. 548-553, 1969.

[2] C. C. Collins, M. R. Carlson, A. B. Scott, and A. Jampolsky, "Extraocular muscle forces in normal human subjects," Investigative Ophthalmology and Visual Science, vol. 20, no. 5, pp. 652-664, 1981.

[3] H. J. Simonsz, "Force-length recording of eye muscles during local anesthesia surgery in 32 strabismus patients," Strabismus, vol. 2, no. 4, pp. 197-218, 1994.

[4] C. Quaia, H. S. Ying, A. M. Nichols, and L. M. Optican, "The viscoelastic properties of passive eye muscle in primates. I: static and step responses," PLoS ONE, vol. 4, no. 4, Article ID e4850, 2009.

[5] Y. C. Fung, Biomechanics: Mechanical Properties of Living Tissues, Springer, New York, NY, USA, 1993.

[6] L. H. Yoo, H. Kim, V. Gupta, and J. L. Demer, "Quasi-linear viscoelastic behavior of bovine extra-ocular muscle tissue," Investigative Ophthalmology and Visual Science, vol. 50, no. 8, pp. 3721-3728, 2009.

[7] C. Quaia, H. S. Ying, and L. M. Optican, "The viscoelastic properties of passive eye muscle in primates. II: testing the quasi-linear theory," PLoS ONE, vol. 4, no. 8, Article ID e6480, 2009.

[8] M. S. Shall, D. M. Dimitrova, and S. J. Goldberg, "Extraocular motor unit and whole-muscle contractile properties in the squirrel monkey: summation of forces and fiber morphology," Experimental Brain Research, vol. 151, no. 3, pp. 338-345, 2003.

[9] S. Sklavos, D. M. Dimitrova, S. J. Goldberg, J. Porrill, and P. Dean, "Long time-constant behavior of the oculomotor plant in barbiturate- anesthetized primate," Journal of Neurophysiology, vol. 95, no. 2, pp. 774-782, 2006.

[10] J. C. Downs, J. K. F. Suh, K. A. Thomas, A. J. Bellezza, R. T. Hart, and C. F. Burgoyne, "Viscoelastic material properties of the peripapillary sclera in normal and early-glaucoma monkey eyes," Investigative Ophthalmology and Visual Science, vol. 46, no. 2, pp. 540-546, 2005.

[11] H. C. Lin, M. K. W. Kwan, and S. L. Y. Woo, "On the stress relaxation properties of the anterior cruciate ligament(ACL)," in Proceedings of the Annual Meeting of the American Society of Mechanical Engineers, pp. 5-6, Advances in Bioengineering, 1987. 
[12] C. E. Miller and C. L. Wong, "Trabeculated embryonic myocardium shows rapid stress relaxation and non-quasilinear viscoelastic behavior," Journal of Biomechanics, vol. 33, no. 5, pp. 615-622, 2000.

[13] V. C. Mow, S. C. Kuei, W. M. Lai, and C. G. Armstrong, "Biphasic creep and stress relaxation of articular cartilage in compression: theory and experiments," Journal of Biomechanical Engineering, vol. 102, no. 1, pp. 73-84, 1980.

[14] I. S. Nash, P. R. Greene, and C. S. Foster, "Comparison of mechanical properties of keratoconus and normal corneas," Experimental Eye Research, vol. 35, no. 5, pp. 413-424, 1982.

[15] J. G. Pinto and Y. C. Fung, "Mechanical properties of the heart muscle in the passive state," Journal of Biomechanics, vol. 6, no. 6, pp. 597-616, 1973.

[16] J. M. Huyghe, D. H. van Campen, T. Arts, and R. M. Heethaar, "The constitutive behaviour of passive heart muscle tissue: a quasi-linear viscoelastic formulation," Journal of Biomechanics, vol. 24, no. 9, pp. 841-849, 1991.

[17] B. S. Myers, J. H. McElhaney, and B. J. Doherty, "The viscoelastic responses of the human cervical spine in torsion: experimental limitations of quasi-linear theory, and a method for reducing these effects," Journal of Biomechanics, vol. 24, no. 9, pp. 811-817, 1991.

[18] S. R. Toms, G. J. Dakin, J. E. Lemons, and A. W. Eberhardt, "Quasi-linear viscoelastic behavior of the human periodontal ligament," Journal of Biomechanics, vol. 35, no. 10, pp. 14111415, 2002.

[19] C. E. Miller, M. A. Vanni, and B. B. Keller, "Characterization of passive embryonic myocardium by quasi-linear viscoelasticity theory," Journal of Biomechanics, vol. 30, no. 9, pp. 985-988, 1997.

[20] S. L.-Y. Woo, B. R. Simon, S. C. Kuei, and W. H. Akeson, "Quasi-linear viscoelastic properties of normal articular cartilage," Journal of Biomechanical Engineering, vol. 102, no. 2, pp. 85-90, 1980.

[21] Y. C. Fung, "Elasticity of soft tissues in simple elongation," The American Journal of Physiology, vol. 213, no. 6, pp. 1532-1544, 1967.

[22] Y. C. Fung, Stress-Strain-History Relations of Soft Tissues in Simple Elongation, Biomechanics: Its Foundations and Objectives, Prentice-Hall, Englewood Cliffs, NJ, USA, 1972.

[23] N. Simin, L. E. Bilston, and N. Phan-Thien, "Viscoelastic properties of pig kidney in shear, experimental results and modelling," Rheologica Acta, vol. 41, no. 1, pp. 180-192, 2002.

[24] G. M. Thornton, C. B. Frank, and N. G. Shrive, "Ligament creep behavior can be predicted from stress relaxation by incorporating fiber recruitment," Journal of Rheology, vol. 45, no. 2, pp. 493-507, 2001.

[25] B. R. Simon, R. S. Coats, and S. L. Y. Woo, "Relaxation and creep quasilinear viscoelastic models for normal articular cartilage," Journal of Biomechanical Engineering, vol. 106, no. 2, pp. 159-164, 1984.

[26] X. Hu and G. S. Daehn, "Effect of velocity on flow localization in tension," Acta Materialia, vol. 44, no. 3, pp. 1021-1033, 1996.

[27] W. Ze-Ping, "Void-containing nonlinear materials subject to high-rate loading," Journal of Applied Physics, vol. 81, no. 11, pp. 7213-7227, 1997.

[28] T. Nicholas, "Tensile testing of materials at high rates of strain," Experimental Mechanics, vol. 21, no. 5, pp. 177-185, 1981. 

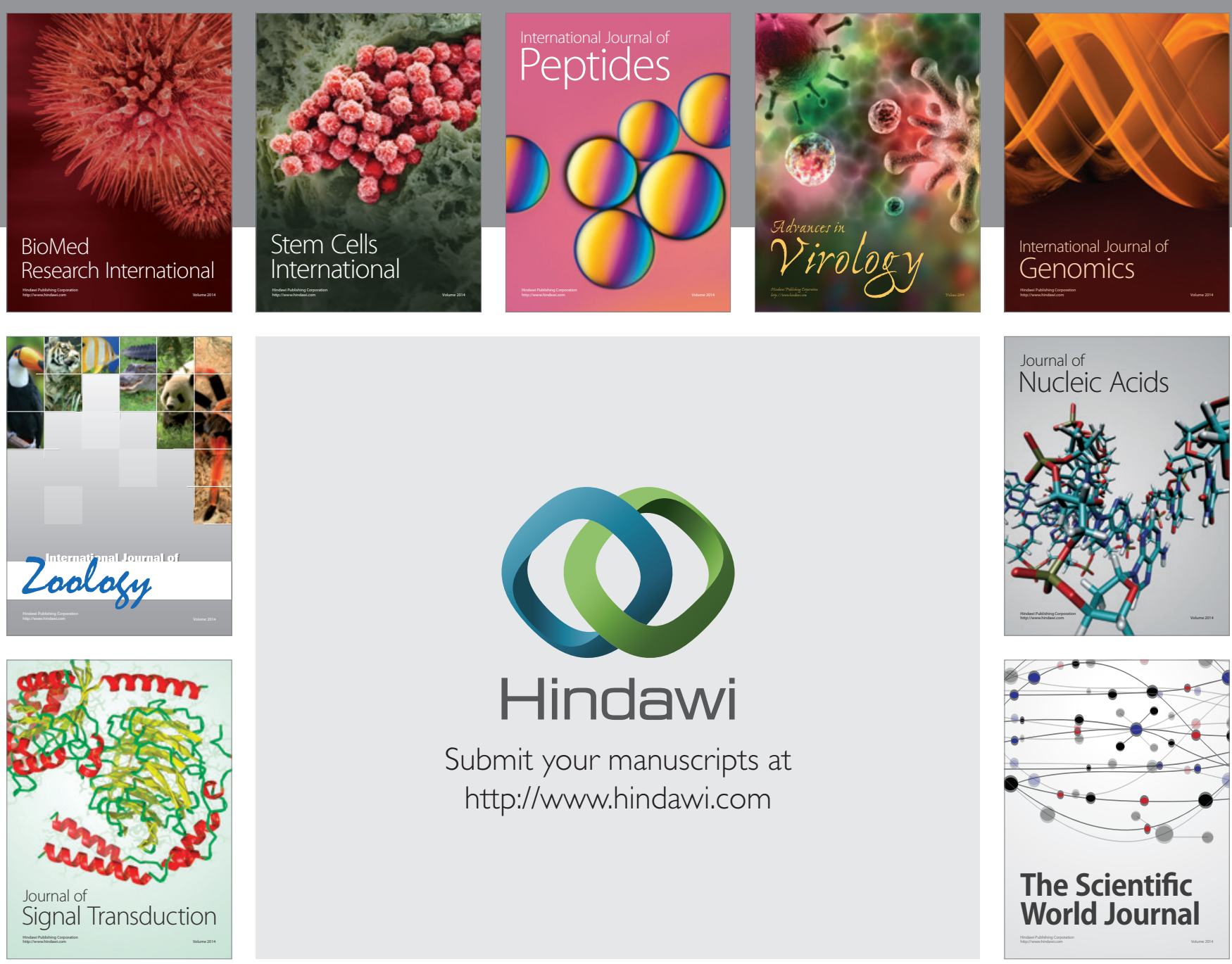

Submit your manuscripts at

http://www.hindawi.com
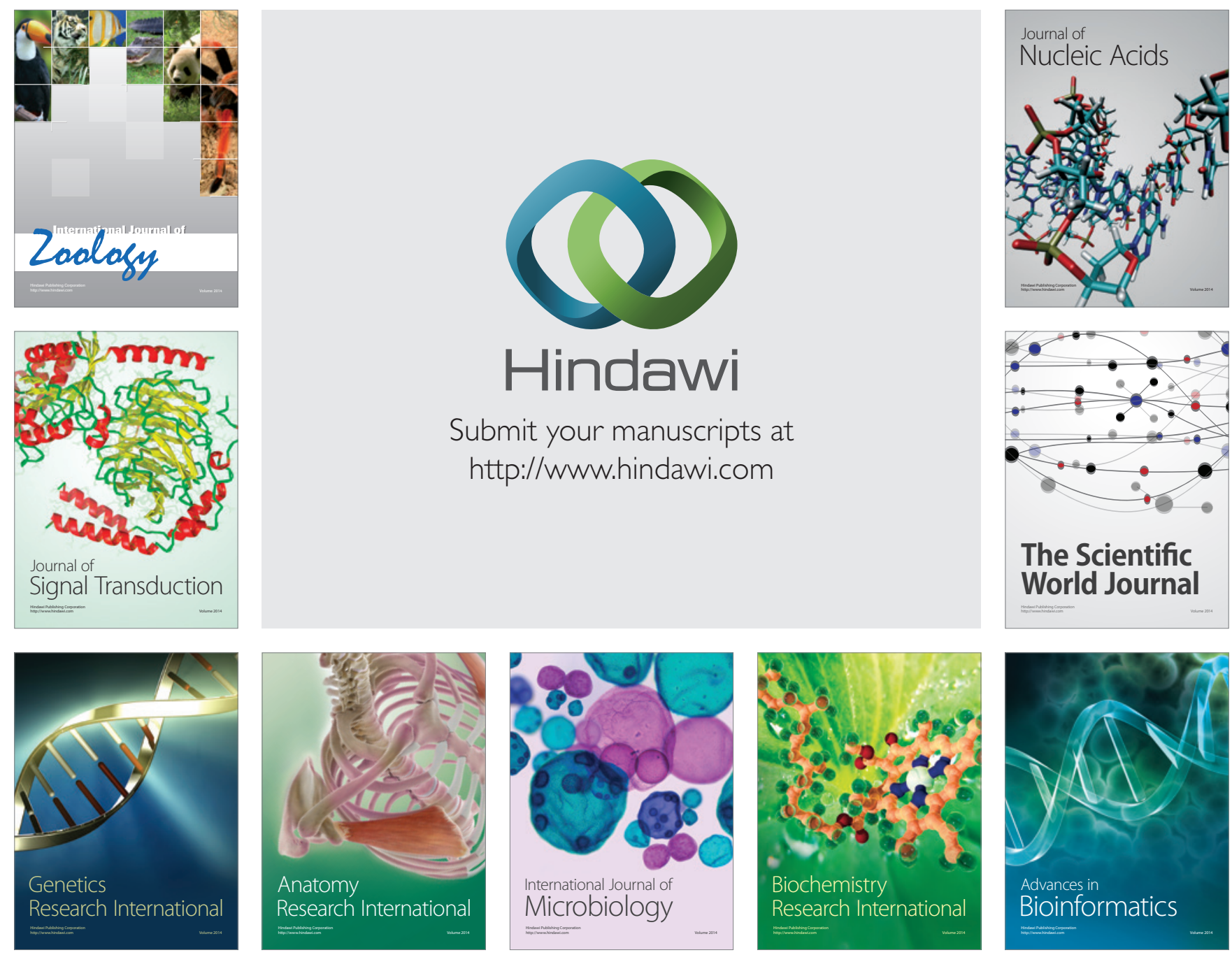

The Scientific World Journal
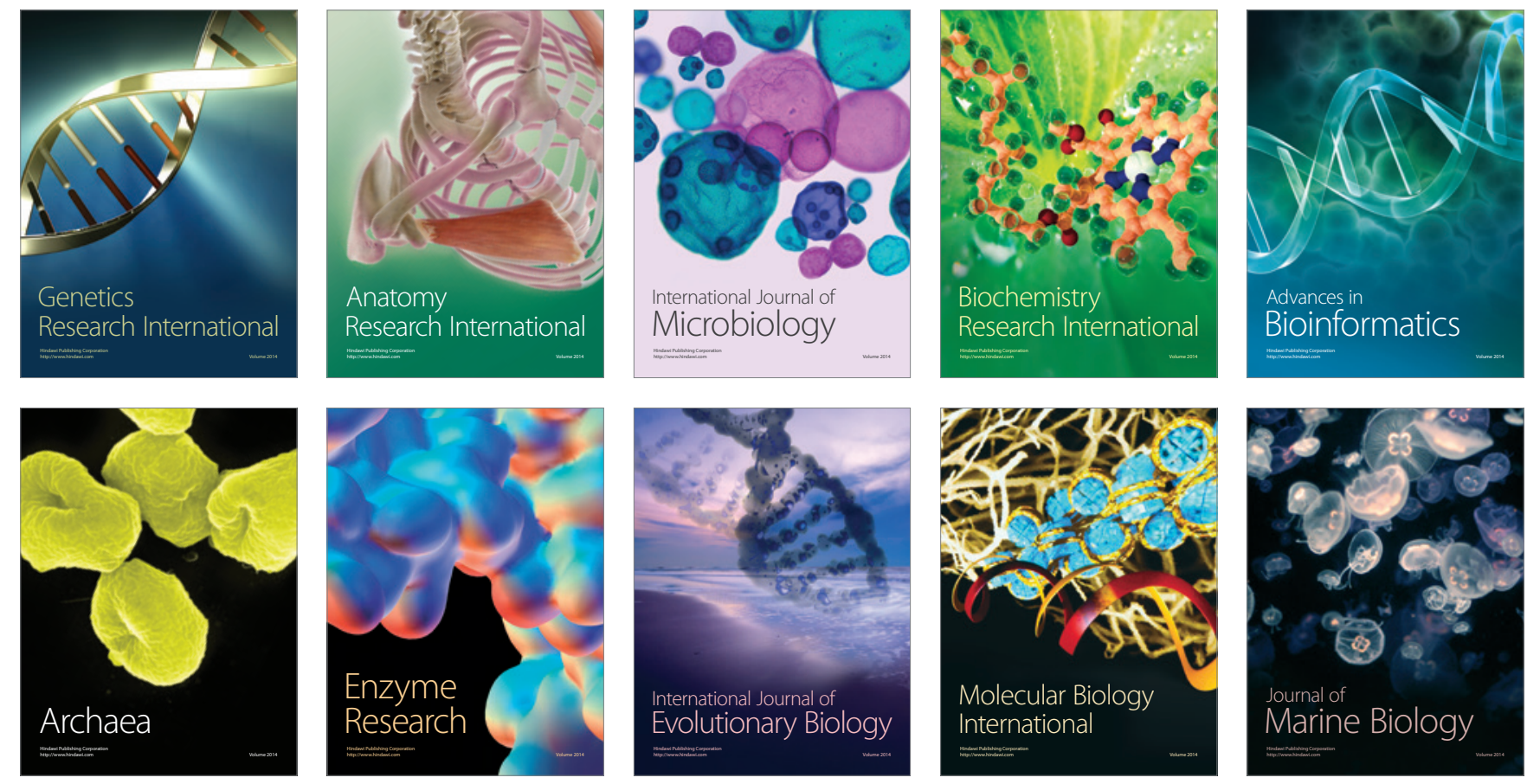